\title{
Functional Characterization of a WD-Repeat Protein Gene (OsWD1) in Rice
}

\author{
Siou-Luan $\mathrm{He}^{1,2,3}$, Shin-Lon $\mathrm{Ho}^{1, \text { * }}$ \\ ${ }^{1}$ Department of Agronomy, National Chiayi University, Chiayi, Taiwan, R.O.C. \\ ${ }^{2}$ Institute of Plant Biology, National Taiwan University, Taipei, Taiwan, R.O.C. \\ ${ }^{3}$ Institute of Plant and Microbial Biology, Academia Sinica, Taipei, Taiwan, R.O.C.
}

\section{Email address:}

slho@mail.ncyu.edu.tw (Shin-Lon Ho)

${ }^{*}$ Corresponding author

\section{To cite this article:}

Siou-Luan He, Shin-Lon Ho. Functional Characterization of a WD-Repeat Protein Gene (OsWD1) in Rice. American Journal of Agriculture and Forestry. Vol. 6, No. 2, 2018, pp. 18-27. doi: 10.11648/j.ajaf.20180602.11

Received: April 7, 2018; Accepted: April 23, 2018; Published: May 11, 2018

\begin{abstract}
A gene encoded WD-40 repeat protein (OsWD1) was isolated from rice cells to study the platform for proteinprotein interactions in germinating seeds. The expression of $O S W D 1$ was found to be specifically induced at $3 \mathrm{~d}$ of seed germination. By ectopic overexpressing $(O s W D 1-O x)$ or RNA interference gene knockdown (OsWD1-Ri) of OsWD1, respectively. Results revealed that the rice seedlings and grain size were enhanced in OsWD1-Ox lines, but were reduced in OsWD1-Ri lines. Moreover, the expression of GA-inducible gene, OsEP3A, which was strongly induced in OsWD1-Ox lines during rice seed germination. By iodine staining in starch containing solid medium, the $\alpha$-amylase activities were further enhanced in $O s W D 1-O x$ lines regardless with or without exogenous GA in the medium. These results suggested that $O s W D 1$ might acts as a platform for protein-protein interactions to regulate seedling and seed development in rice by a GA-dependent manner.
\end{abstract}

Keywords: Rice, OsWD1, Overexpression, Plant Height, Grain Size, Gibberellin Acid

\section{Introduction}

The WD-repeat proteins, consisting of a diverse superfamily of regulatory proteins, were originally identified from $\mathrm{G}$ protein $\beta$ subunit, termed as $\beta$-transducin repeats [1]. Many paralogs and orthologs of WD-repeat proteins in plants and animals have been reported, and their functions have been identified to serve as a platform for protein-protein interaction [2-4]. The WD (Trp-Asp) motif comprising about 40 - 60 residues, are initiated by the GH (Gly-His) dipeptide of 11-24 amino acids from its $\mathrm{N}$-terminus and typically ends with the WD dipeptide at the C-terminus, but neither the GH dipeptide nor the WD dipeptide is absolutely conserved, and the WD motif is usually found as $4-16$ tandem repeats within a single protein $[1,5]$. The WD-repeat was marked by the characteristic core element of $(\mathrm{G}, \mathrm{V}, \mathrm{A}, \mathrm{N}, \mathrm{S}) \mathrm{H}-\mathrm{X}_{28}-(\mathrm{W}$, F) D, where $X$ stands for any amino acid [1] (Figure 1b). It seems that the WD-repeat units would fold into a higherorder conformation and form a $\beta$-propeller, and this structure is thought to serve as stable platforms for the assembly of protein complexes or mediators of transient interaction with other proteins $[2,4-7]$. The WD-repeat large family proteins have been found only in eukaryotes and can be divided into several subfamilies. These members play important roles in the regulation of a wide variety of biological processes, such as signal transduction, light signaling, transcriptional regulation, cell cycle and cytokinesis, mRNA processing and protein trafficking $[1,2,5,8]$. WD-repeat proteins have also been found to be involved in flowering, anthocyanin biosynthesis, mitochondrial fission, and chromatin assembly [9-12]. Such an astonishingly functional diversity may be explained by the potential role of WD-repeat-related proteins that can interact with several proteins via coordinating sequential and/or simultaneously facilitating the formation of multiprotein complexes $[5,7]$.

In plants, several WD-repeat proteins have been isolated showing a wide variety of biological functions. In Arabidopsis, AGB1 (Arabidopsis heterotrimeric G-protein $\beta$ 
subunit) encodes a protein that shows significant similarity to animal $\mathrm{G}$ protein $\beta$ subunits. Mutation of agbl in Arabidopsis shows several developmental defects, including short, blunt fruits, rounded leaves, and shortened floral buds. Thus, it was proposed that AGB1 played the role of a plant developmental regulator through the heterotrimeric G-protein signaling [9]. Another well characterized WD-repeat protein, COP1, from Arabidopsis, is essential for plant development and function as a negative regulator of photomorphogenesis during seedling growth [13-15]. In tomato, LeMSI1 [16], encodes a WD-repeat protein orthologs of human RB (retinoblastoma)-binding proteins, RbAp48 [17] and RbAp46 [18], and yeast Msi1 [19]. These proteins are thought to form a protein complex, and are involved in the negative regulation of the cell cycle, histone acetylation and chromatin assembly. Protein-binding activity analysis has shown that LeMSI1 can interact with human RB, suggesting that it plays a potential role in cellular growth regulation [16]). In yeast, $P f s 2 p$ encodes a novel regulatory WD-repeat protein, which was predicted to participate in the pre-mRNA 3'-end processing by bridging different processing factors, and thereby promoting the formation of the multiprotein processing complex [20]. A well known Arabidopsis WDrepeat protein, PRL1, is an SNF1 homologs-binding protein that shows glucose-dependent interaction with yeast SNF1 and Arabidopsis AKIN10 and AKIN11, mutation of the PRL1 gene, which results in glucose hypersensitivity and derepression of glucose-regulated genes, indicating PRL1 functions as a negative regulator of Arabidopsis SNF1 homologs [21]. Overexpression of PFWD (Perilla frutescens WD-repeat protein) in Arabidopsis showed an enhanced anthocyanin accumulation in aerial parts and reduced viability, suggesting that $P F W D$ plays a role in the regulation of anthocyanin biosynthesis [22]. In yeast, Mdv1p containing seven WD-repeat units at the C-terminal regulatory domain, acts as a molecular adaptor to regulate the interaction between Dnm1p and Fis1p, and catalyzes a rate-limited step in mitochondrial fission $[10,23]$.

There are about 200 potential WD-40 repeat genes in rice [24]. Among which the ASP1 (Aberrant Spikelet and Panicle 1), OsLIS-L1 (Lissencephaly type-1-like 1), and OsREL2 (Ramosal Enhancer Locus 2) encodes the same protein (Os08g0162100). The aspl mutant showed derepression of axillary bud growth and disturbed phyllotaxy in the vegetative phase; osrel2 mutant showed several developmental defects, such as defects in panicle heading, sterile lemma elongation, and panicle development; and oslis-ll mutant showed abnormal developmental phenotypes, including semi-dwarf, shorter panicle length, and reduced male fertility [25-27]. Mutation of this gene in rice showed similar phenotypes that indicated an important role on the regulation of vegetative and reproductive development. This study focus on the functional characterization of $O s W D 1$, a member of WD-40 family in rice, its gene expression was induced in germinating embryos and senescing leaves. Based on gene overexpression and double strand RNA gene silencing strategies, we demonstrated that $O s W D 1$ might play a positive role on regulation of rice seedling growth and seeds development.

\section{Materials and Methods}

\subsection{Materials}

The rice variety used in this study was Oryza sativa L. cv Tainung 67 (TNG 67). Immature seeds were dehulled, sterilized with $2.4 \% \mathrm{NaOCl}$ for $30 \mathrm{~min}$, washed extensively with sterile water, and placed on N6 solid medium containing 2, 4-D (2.0 mg/L) for callus induction. After 1 month, callus derived from scutellum was transferred to a liquid Murashige and Skoog (MS) medium [28] containing 3\% Sucrose and 10 $\mu \mathrm{M} 2,4-\mathrm{D}$ to establish a suspension cell culture, or subcultured in fresh N6D agar medium for Agrobacteriummediated gene transformation. The cell suspension was cultured on a reciprocal shaker at $120 \mathrm{rpm}$ and incubated at $26^{\circ} \mathrm{C}$ in the dark.

\subsection{DNA and Protein Sequence Analysis}

The DNA sequence of OsWD1 was compared with the GenBank database using Blastn program. Information and homology of amino acid sequence was analyzed using Blastp at NCBI (http://www.ncbi.nlm.nih.gov/blast). Prediction of WD40 repeat domains and tertiary structure was done with the e-mail server at the BMERC (http://bmercwww.bu.edu/wdrepeat/).

\subsection{Primers}

Sequences of all primers used for PCR amplification of DNA fragments are listed in Table 1.

Table 1. Primers and their sequences used in this study.

\begin{tabular}{ll}
\hline Name & Sequence \\
\hline OsWD1-F1 & 5'-ACTCTCGAGATGAGGAGAAGTTCAAGGAG-3' \\
OsWD1-R1 & 5'- CTACTCGAGTTATCTTTCTGGTTGATCAGAA-3' \\
OsWD1-F2 & 5'-ACTGGATCCGATCAACCAGCGCTTTGTCC-3' \\
OsWD1-R2 & 5'-ACTCTGCAGAGCAGCAATTTACTTTCTAGTT-3' \\
GFP-F3 & 5'- TATCTGCAGGTGAGCAAGGGCGAG-3' \\
GFP-R3.1 & 5'- TCTGTCGACTTACTTGTACAGCTCGTC-3' \\
GFP-R3.2 & 5'- TCTGTCGACACGCTGAACTTGTGGC-3' \\
\hline
\end{tabular}

Gene accession number: OsWD1 (AK111518); GFP (U43284)

\subsection{Construction of the Expression Vectors}

To construct the vector for ectopic expression of OsWD1 in transgenic rice, a 3357-bp DNA fragment that contained the complete coding region of OsWD1 was amplified using the $O s W D 1-O x$ forward primer (OsWD1-F1) and OsWD1-Ox reverse primer (OsWD1-R1). This DNA fragment was digested with XhoI, blunt-ended the restriction sites and cloned into the blunt-ended BamHI site of the pAHC18 expression vector [29]. This recombinant plasmid was linearized by partial digestion with PstI and inserted into the Pst I site of the pSMY1H binary vector [30] for the Agrobacterium-mediated gene transformation.

To construct the OsWD1 RNAi vector, a 405-bp DNA 
fragment containing $340 \mathrm{bp}$ of the 3'-untranslated region and 65 bp of the 3 '-end coding region of OsWD1 was amplified using the $O s W D 1-R i$ forward primer (OsWD1-F2) and OsWD1-Ri reverse primer (OsWD1-R2). This DNA fragment was digested with BamHI and PstI and cloned into the BamHI/Pst I sites of pBluescript, generating pWK1. A truncated form of green fluorescent protein (GFP) cDNA was amplified by PCR using the forward primer (GFP-F3) and reverse primer (GFP-R3.1). The resulting PCR product was digested with PstI and SalI and inserted into the PstI/SalI sites of the pWK1, generating pWK2. A 495-bp DNA fragment containing $405 \mathrm{bp}$ of the 5'-end of OsWD1 DNA and $90 \mathrm{bp}$ of the 5' sequence of GFP was amplified by PCR using the forward primer OsWD1-F2 and reverse primer GFP-R3.2. This DNA fragment was digested with $K p n \mathrm{I}$ and $S a l \mathrm{I}$ and cloned in the antisense orientation into the $S a l \mathrm{I} / \mathrm{KpnI}$ sites of pWK2, generating pWK3. A 1,620-bp DNA fragment comprising OSWD1-GFP in both the sense and antisense orientations was obtained from pWK3 by digestion with BamHI and ligated into the BamHI site of the AHC18 expression vector, generating pAHC18-OsWD1-Ri. This RNA silencing construct was linearized by digestion with HindIII and inserted into the HindIII site of the pSMY1H binary vector for the Agrobacterium-mediated gene transformation.

\subsection{Plant Transformation}

The expression vectors were introduced into Agrobacterium tumefaciens strain EHA101 by electroporation. The rice plants were transformed as described previously [30]. Calli induced from immature rice embryos were cocultured with $A$. tumefaciens. Transformed calli were selected on N6 medium that contained $50 \mathrm{mg} / \mathrm{L}$ hygromycin B.

\subsection{Southern Blot Analysis of Transgenic Rice Plants}

Genomic DNA was isolated from the suspension-cultured cells of rice. Ten micrograms of genomic DNA was digested with restriction enzymes, fractionated in a $0.8 \%$ agarose gel, and transferred to a nylon membrane. Hybridization was performed at $42^{\circ} \mathrm{C}$ using ${ }^{32} \mathrm{P}$ random-primer-labeled full length of $O s W D 1$ coding region (3357 bp) (Figure 3) or hygromycin phosphotransferase gene (Figure $4 \mathrm{~b}$ ) as a probe.

\subsection{Northern Blot Analysis of Transgenic Rice Plants}

Total RNA was isolated from various tissues of germinating seeds and from suspension-cultured cells using TRIzol reagent (Invitrogen). The RNA gel-blot analysis was performed as described [31]. Ten micrograms of total RNA was electrophoresis in $1 \%$ agarose gel containing $10 \mathrm{mM}$ sodium phosphate buffer $(\mathrm{pH}$ 6.5), transferred to a GeneScreen Plus (NEN/Dupont, Wilmington, DE) membrane, and hybridized at $42^{\circ} \mathrm{C}$ with ${ }^{32} \mathrm{P}$ random-primerlabeled gene-specific probe, which was prepared from the 3'untranslated region of OsWD1 (405 bp) (AK111518, the nucleotide sequence from 3741-4145 bp).

\subsection{RT-PCR Analysis of Gene Expression}

The expression levels of $O S E P 3 A$ in transgenic rice were evaluated by RT-PCR. Total RNA isolated from embryos of $4 \mathrm{~d}$ germinated seeds was used to synthesize first-strand cDNAs using oligo(dT) primers, which were subjected to PCR analyses using the primers OsEP3A-RT5 and OsEP3ART3. PCR products were resolved by agarose gel electrophoresis.

\subsection{Iodine Staining in Starch Containing Solid Medium}

The middle of rice seeds were cross cut with a razor blade after dehulled and sterilized. The half-seeds (with embryo) were placed onto the starch agar $(0.2 \%$ potato starch, $5 \mathrm{mM}$ $\mathrm{CaCl}_{2}, 20 \mathrm{mM}$ sodium succinate $\mathrm{pH} 5.0,0.4 \%$ phytagel) plates, in which were supplemented with or without $10 \mu \mathrm{M}$ $\mathrm{GA}_{3}$, respectively. After incubated for $3 \mathrm{~d}$, the plate was flooded with $2 \mathrm{~mL}$ iodine solution $\left(0.1 \% \mathrm{I}_{2}, 1 \% \mathrm{KI}\right)$, if a clear area around the half-seed will appear, indicated where starch had been breakdown into glucose by the $\alpha$-amylase activity.

\section{Results}

\subsection{Sequence Analysis of OSWD1}

To identify rice genes that are act as a platform for proteinprotein interactions in germinating rice seeds. The OSWD1 (AY111518), which encodes a putative protein of 1133 amino acids with an estimated molecular mass of $125 \mathrm{kDa}$, was selected for further study. After analyzed with the Conserved Domain Database [32] and EMBL SMART (Simple Modular Architectur Research Tool) database [33], we found that OsWD1 contains an $\alpha$-helical structure of CTLH motif (Cterminal to LisH motif) at the $\mathrm{N}$-terminal (Figure 1a, residues 34-92), and exhibits ten WD-repeat motifs at the C-terminal, in which five of WD-repeat units between amino acids 354 and 662 , the other of five repeats located between amino acids 821 and 1094 (Figure 1a). Sequence comparison of the ten WD-repeat units in OsWD1 revealed that each of the WD-repeat comprises a typically consensus residues (Figure 1b), which was compiled for about 900 potential WD-repeat units from 152 WD-repeat proteins [5]. The 3-D structure of OsWD1 protein was also predicted by BMERC (http:// bmerc-www.bu.edu/wdrepeat/), each of the WD-repeat unit formed a four-stranded (a blade), antiparallel $\beta$-sheet, in which the $\beta 1$-strand is closest to the central pore and $\beta 4$ strand forms the outer surface of the folded protein (Figure 1b) [5]. There are ten blades within the OsWD1 protein that fold into a higher-order structure known as $\beta$-propeller (Figure 1c), suggesting that it serves as a platform for protein-protein interaction. There is a sequence stretch about 159 amino acids located between the first and the second five WD-repeat units (Figure 1a), suggest that the ten WD-repeat domains may be divided into two smaller propellers other than one large propeller. 
A

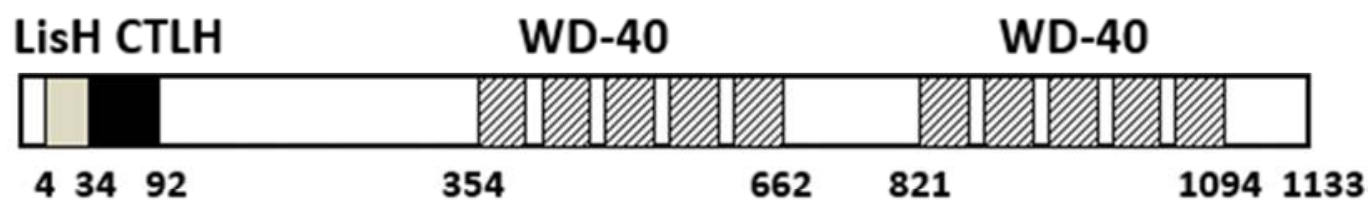

B

\begin{tabular}{|c|c|c|c|c|c|c|c|c|c|c|}
\hline & & Varial & e region & & & Core regio & & & & \\
\hline & & $\beta 4$-strar & & & $\beta 1-s t$ ran & & 2-strand & & 3-strand & \\
\hline $\mathrm{m}$ & 382 & rosul & QHSLI [?] & DPTSST & MoIres & FDGT & LFGAI & SPII & IFQIIS & 424 \\
\hline II & 425 & IOGDD & IMQIIEID & AIIGGT & MDLAFA & IPTQL. & cImळ & $\mathrm{DDr}$ & TIKFE & 471 \\
\hline$=113$ & 472 & $\Delta \operatorname{ASCAX}$ & QFIFE & GIBAPT & एकाक & ImIQ & FIPSTS & LDG & IIAE. & S15 \\
\hline W4 & S16 & IILCS & RTID & APGIXC & muss & $\triangle D C S R L$ & FSOTS & IIG & EIIIE & 559 \\
\hline mS & 560 & DESEG & AHETIDG & FRTS & GFD & $\pi \mathrm{m}$ & PFAAG & $\mathrm{DE}$ & LXIV & 604 \\
\hline W6 & 89 & PSILM & TOITD [?] & XPEDA & ICFAIS & nas & rinses & $G \mathbb{X}$ & ISLPM & 851 \\
\hline m? & 852 & MIrIm & $\operatorname{Tr} x$ & PPPPA & TrLAFI & PQIOO & IIAICM & $\mathrm{DIS}$ & IQIm & 893 \\
\hline m8 & 894 & IRIDET & KSILR & GISTII & TGLATS & XWD & IIISSG & Ant & QICHS & 935 \\
\hline m9 & 936 & THGTI & LESTாஹIPSS & RPSSII & LITR & nQpDI & FLTIE & $\mathrm{IQI}$ & AIIEII & 986 \\
\hline m10 & 987 & IIEPT & QPPTR & EXSPI & nEXS & asp & LIIASF & Lns & тести & 1028 \\
\hline Consens & & EFHF & $\mathbf{x}[1-?]$ & GXHI & Itxx & $\mathbf{x}[0-?] P D G[0-3]$ & Insas & XII & TIEFE & \\
\hline & & & & $\begin{array}{ll}\mathbf{A} & \mathrm{I}\end{array}$ & L 1 & SXS & IFIAG & & STRLPI & \\
\hline & & & & & I L & ISP & II. SA & & L IY & \\
\hline & & & & & C I & & F C & & & \\
\hline
\end{tabular}
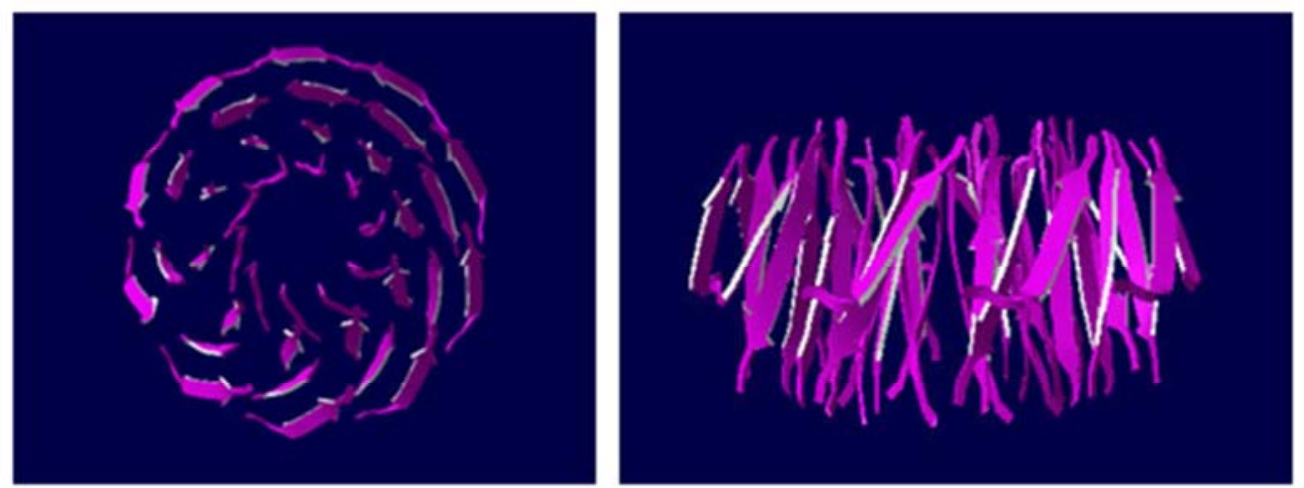

Figure 1. Schematic diagrams and consensus sequence analysis of WD-repeat units in OsWD1. (A) OsWD1 consists of 1133 residues and contains ten WDrepeat motifs and a CTLH (C-terminal to Lish motif) $\alpha$-helical motif at the $N$-terminal region. (B) Comparison of the ten WD-repeat units in OsWD1. Arrows indicate that each WD-repeat unit contains a variable and core regions according to Neer et al. [1]. The core regions start with GH and end with WD that marked with the red letters. The blue color regions are predicted to fold into the $\beta$-strand. A consensus sequence compiled for 152 WD-repeat proteins is shown for comparison [5]. X indicates that any amino acid can be found in that position. The [?] signifies that this variable region is still unidentified. (C) Left panel: structure of the WD-repeat domains (top view). The tertiary structure prediction was carried out with the e-mail server at the http://bmercwww.bu.edu/wdrepeat. The generated core model coordinates for the ten WD-repeat units as a beta propeller. Right panel: structure of the WD-repeat domains (side view).

By searching the NCBI database, there are two unidentified of OsWD1 homologous genes are found, OsWD2 (Os08g0162100) and OsWD3 (Os01g0254100) in rice genome, which share $76 \%$ and $71 \%$ amino acid identity, respectively. Blastp search (Figure 2) revealed that there are several closest homologs to OsWD1 in different plant species, as described in the following: TOPLESS from
Arabidopsis thaliana (80\% amino acid identity); an unknown function of WD40 repeat protein from maize (93\% amino acid identity); CTV.2 from Poncirus trifoliate (82\% amino acid identity), a putative WD40 repeat protein predicted in the Ctv region, Ctv was physically mapped to a $282-\mathrm{Kb}$ region that confers the broad spectrum resistance to the major virus pathogen, CTV (Citrus tristeza virus), in citrus [34]. 


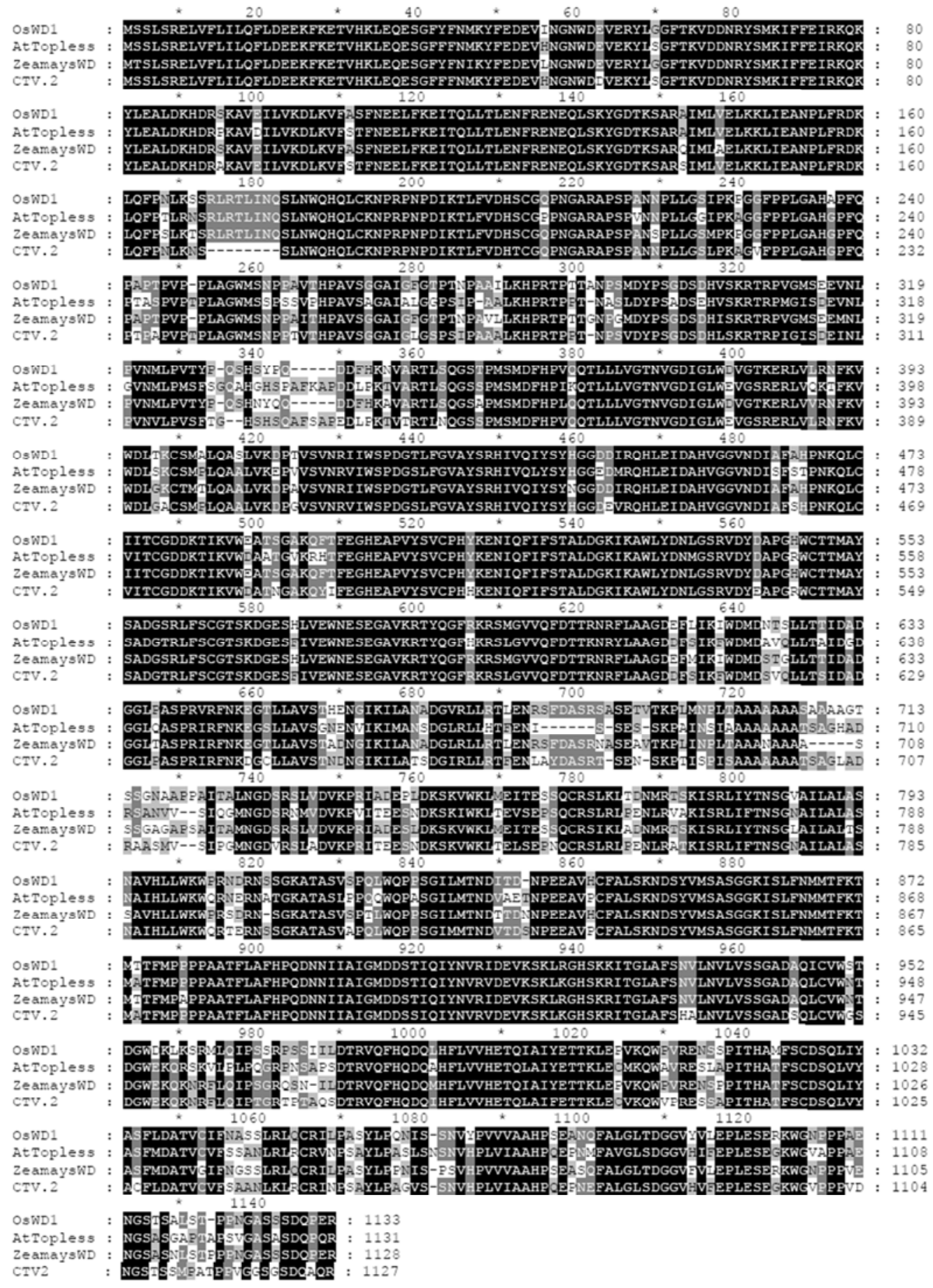

Figure 2. Multiple sequence alignment of OsWD1 homolog proteins. OsWD1 (AK111518), a WD-repeat protein in rice; AtTOPLESS (At1g15750), ZeamaysWD (BT061468), an unknown function protein in maize; PtCTV2 (AAN62336), a CTV.2 protein in Poncirus trifoliate. Black boxes indicate completely identical amino acids; residues conserved across three or four sequences are shaded gray. The numbers indicate the positions of the amino acids within the individual proteins. Dots indicate gaps in the sequences to allow maximal alignment. Alignment was performed by the Pileup program of the University of Wisconsin Genetics Computer Group software package; shading of conserved residues was accomplished with the GeneDoc program.

\subsection{Temporal Expression of OsWD1 in Germinating Rice Embryo}

To examine the expression level of $O S W D 1$ during rice seed germination, total RNA was purified from various time periods of germinating rice embryo and subjected to northern blot analysis. As shown in Figure 3, the accumulation of OsWD1 mRNA was not detectable in the embryos of dry seeds or one day after imbibition (Figure 3, lane 1 and 2), and then became detectable on day 2 (lane 3 ) and increased to the highest level on day 3 (lane 4), then gradually decreased from day 4 to day 5 (lanes 5 and 6), and became barely detectable after day 6 (lane 7-11). These results demonstrate that the expression of $O s W D 1$ in rice plant is developmentally regulated. 


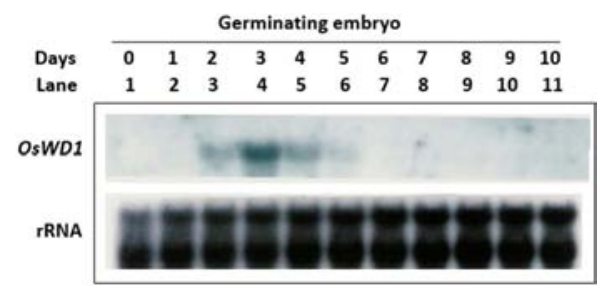

Figure 3. The gene expression levels of OsWD1 in germinating rice seed. Rice seeds were germinated for various time periods. Total RNA was purified from the embryos and subjected to northern blot hybridization using the OsWD1 3'-untranslated region as a probe.

\subsection{Ectopic Expression of the OsWD1-Ox and the OsWD1-RNAi in Transgenic Rice}

To understand the physiological role of OsWD1, both lossand gain-of-function approaches were conducted. A full length of OsWD1, which encodes 1133 amino acid residues was fused downstream of the maize ubiquitin gene ( $U b i)$ promoter (Figure 4a) and transformed into rice callus by agrobacteriummediated gene transformation, to produce the gene overexpression of $O s W D 1$ in transgenic rice (OsWD1-Ox). To generate OsWD1-knockdown transgenic rice, the doublestranded RNA gene-silencing method was used. An inverted repeat of the 3' end 405-bp specific region of OsWD1 was fused up- and down-stream of a truncated GFP coding sequence, and the fusion construct was expressed under the control of the maize $U b i$ promoter, thereby introduced into rice callus by agrobacterium, to create the OsWD1 geneknockdown (OsWD1-Ri) of transgenic rice plants (Figure 4a). Eight independent transgenic lines were randomly selected from both transgenic constructs, respectively, followed by Southern blot hybridization to verify the copy number in those independent transgenic lines. After hybridization with the probe prepared from antibiotic resistance gene, hygromycin phosphotransferase $(\mathrm{Hpt})$, there were at least 5 independent lines exhibited single copy of transgene in both OsWD1-Ox and $O s W D 1-R i$ transgenic plants, respectively (Figure 4b). For northern blot hybridization, total RNA was extracted from five and four independent T2 transgenic plants of OsWD1-Ox and OsWD1-Ri, respectively, and the expression levels of OsWD1 mRNA were examined after hybridized with the specific probe prepared from OsWD1 3'-untranslated region (405-bp). The OsWD1 mRNA was highly expressed in all OsWD1overexpressing lines (Figure 4c, left panel), whereas the endogenous OsWD1 mRNA was barely detectable in three OsWD1-Ri transgenic lines (Figure 4c, right panel). The OsWD1 knockdown line $R i-1$ and $R i-2$, and OsWD1overexpressing line $O x-1$ and $O x-2$ were selected for further study.

A

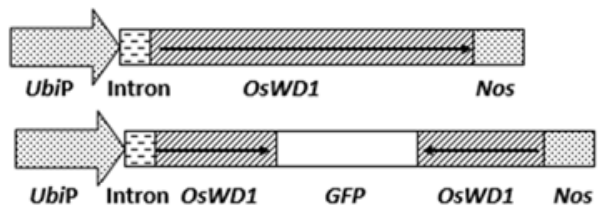

B

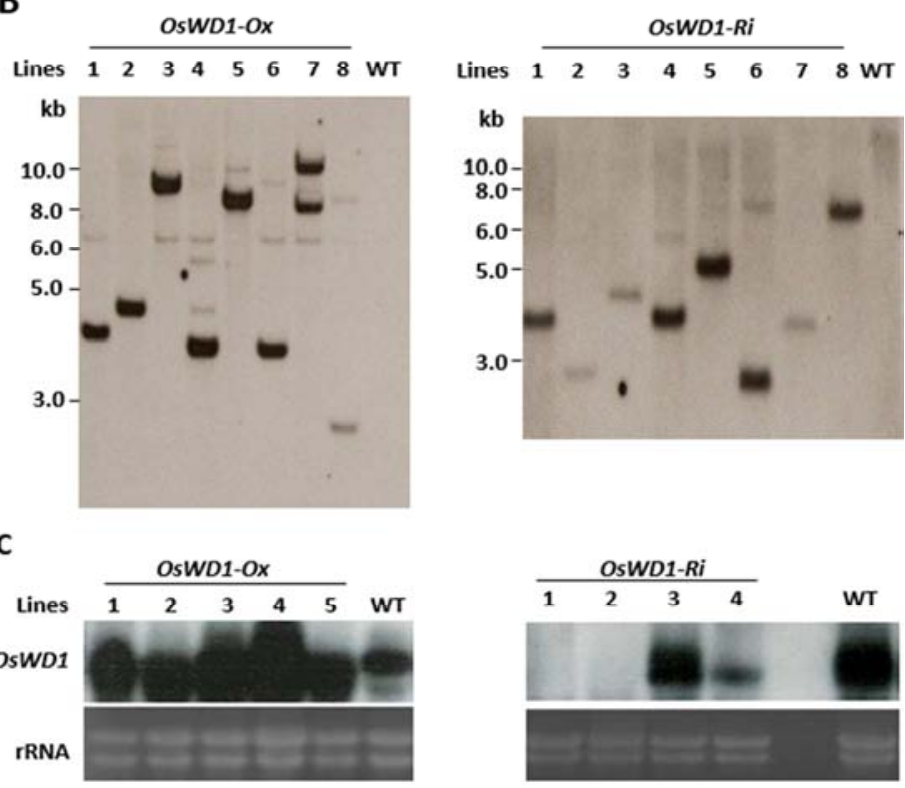

Figure 4. Ectopic expression of OsWD1-Ox and OsWDI-Ri constructs in transgenic rice. (A) Schematic diagram of the overexpression of OsWD1(OsWD1$O x)$ and the double strand RNA gene silencing (OsWD1-Ri) constructs. (B) Genomic DNA gel-blot analyses to identify the copy number of transgene in transgenic lines. The T1 transgenic rice genomic DNA was isolated from leaves of the OsWD1-Ox and the OsWDI-Ri lines, and digested with PstI and subjected to gel-blot analysis using hygromycin phosphotransferase gene (Hpt) as a probe. (C) Expression of OsWD1 in T2 transgenic rice seedlings. Total RNA was isolated from 14-day-old seedlings and subjected to Northern blot analysis using the OsWD1 3'-untranslated region as a probe. Ethidium bromide staining detected $28 S$ and $18 S$ rRNA bands. OsWD1 overexpressing lines containing OsWD1-Ox-1, -2, -3, -4, and -5; OsWD1 knockdown lines containing OsWD1-Ri-1, -2, -3 and -4; wild-type (WT). 


\subsection{The Seedling Lengths and Seed Size is Increased by Overexpression (OsWD1-Ox) and Reduced by Gene Knockdown (OsWD1-Ri) of OsWD1 in Transgenic Rice}

The differences in plant heights of $\mathrm{T} 1$ transgenic lines were observed, with $O s W D 1$ overexpressing lines being taller, whereas $O s W D 1-R i$ transgenic lines being shorter than wildtype plants (Figure 5a, left panel). Elongation of 14-day-old $O x-1$ seedlings was inhibited by uniconazole (Figure 5 a, right panel), indicating that the taller phenotype of $O x-1$ was resulted from over-production of GA. The plant height was further compared between wild-type and T2 transgenic plants. Plant heights of 14-day-old $O x-1$ transgenic seedlings were $30.5 \%$ taller, whereas Ri-1 seedlings were $29 \%$ shorter, than wild-type plants (Figures $5 \mathrm{a}$ and $5 \mathrm{c}$ ). The grain size of the $O x$ 1 and $O x-2$ lines were larger than that of wild type. In contrast, the grain size of $R i-1$ and $R i-2$ lines were smaller than that of wild type (Figure $5 \mathrm{~b}$ ). In addition, the 1,000-grain weight increased by $9.1 \%$ in the $O x-1$ line, but decreased by $15.6 \%$ in the $R i-1$ line, as compared with the wild type (Figure 5d). These results demonstrate that the seedling growth and grain size are positive affected by $O s W D 1$ in rice.
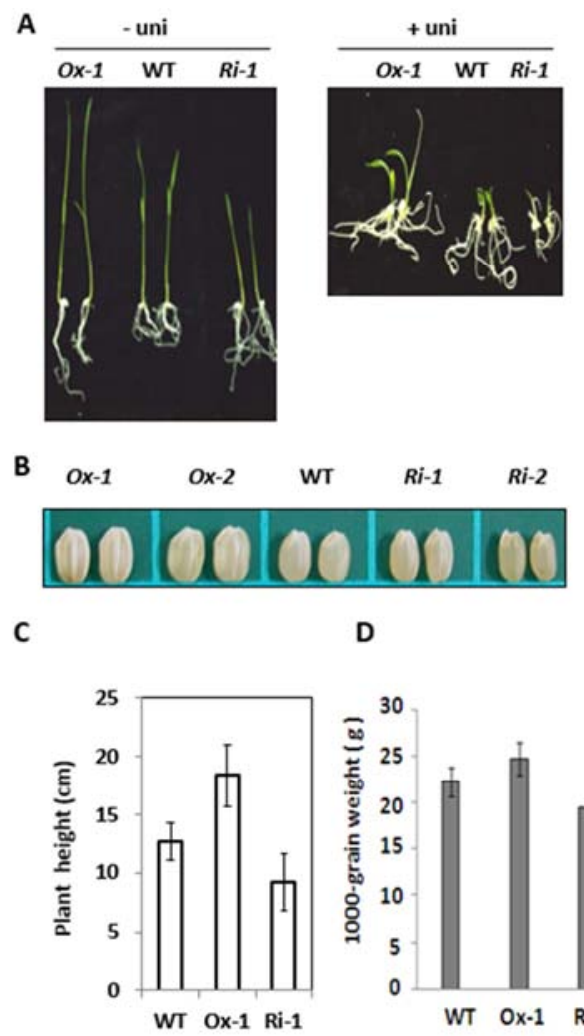

Figure 5. Plant height and seed size in transgenic rice were increased by OsWD1-Ox and reduced by OsWD1-RNAi in transgenic rice. (A) Phenotypes of WT, OsWD1-Ox, and OsWD1-Ri seedlings. Seedlings were grown in 1/2 MS medium for 14 days. (B) Comparison of grain size between WT, OsWD1Ox, and OsWD1-RNAi. Two seeds per line were positioned in a row and photographed together. (C) Quantification of plant height at seedling stages. The plant height of 14-day-old seedling was measured. Error bars indicate standard errors of ten individual plants for each line. (D) Comparison of grain weight of T2 OsWD1-Ox and OsWD1-Ri lines with wild type (WT). Grain weights were calculated from five independent transgenic lines.

\subsection{OsWD1 Enhanced the Expression of GA-Inducible Genes, OsEP3A and o-Amylase}

To confirm the higher length in $O s W D 1-O x$ seedlings are correlated with GA-regulated pathway. The expression levels of a rice cysteine protease gene, $O s E P 3 A$, that is known to be inducible by GA in germinating embryos [30], was determined. Total RNA was isolated from rice embryos germinated for $4 \mathrm{~d}$ and subjected to RT-PCR analyses. As shown in Figure 6a, the abundance of OSEP3A mRNA was increased in embryos of $O x-1$ and $O x-2$, but reduced in Ri-1 and Ri-2 as compared with the wild-type. These studies demonstrated that the expression of $O S E P 3 A$ in germinating embryo is enhanced by $O S W D 1$. Moreover, the well know GA-inducible gene in germinating rice seed, $\alpha$-amylase, was also examined by iodine staining of starch. The mature rice seeds from WT, OsWD1-Ox and $O s W D 1-R i$ were cross cut in the middle with a razor blade, and the half-seeds (with embryo) were placed onto the starch agar plates, in which were supplemented with or without $10 \mu \mathrm{M}$ $\mathrm{GA}_{3}$, respectively. After $3 \mathrm{~d}$ of incubation, the plate was stained with iodine solution, if a clear area surrounding the half-seed was observed, indicated where starch had been breakdown into glucose by the $\alpha$-amylase activity. As shown in Figure 6b, the clear area around each of half-seed was larger in OsWD1-Ox and smaller in OsWD1-Ri than that of the WT. These results demonstrated that the role of OsWD1 in increasing rice seedling growth might through a GA-dependent regulation pathway.

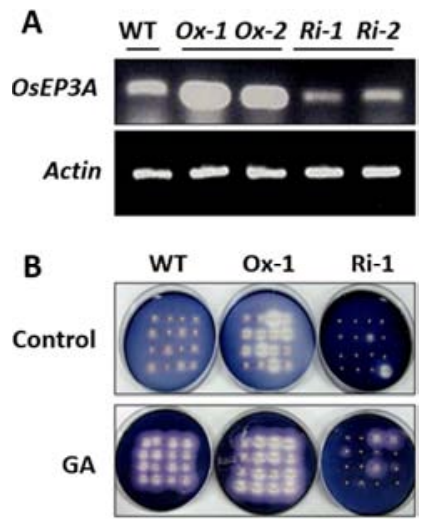

Figure 6. The expression of GA-inducible genes, OsEP3A and $\alpha$-amylase, were enhanced by OsWD1. (A) RT-PCR analysis the expression level of OsEP3A in the OsWD1-Ox and the OsWD1-Ri lines during seed germination. Total RNA was isolated from the embryos of $4 d$ germinated seeds and subjected to RT-PCR analyses. Rice Actin 1 was used as a loading control. (B) Detection of $\alpha$-amylase activity in the OsWD1-Ox and the OsWD1-Ri lines by iodine staining of starch. The rice seeds were cross cut in the middle and the half-seeds (with embryo) were placed onto the starch agar plates. After $3 d$ of incubation, the plates were flooded with iodine solution, a clear area surrounding the half-seed will appear where the $\alpha$ amylase activity occurred.

\section{Discussion}

Many studies have reported that WD-40-related proteins play a key role in GA signaling and biosynthesis pathways in plant developmental processes [35-37]. In the present study, 
OsWD1 is temporal and transient expression at early stages of seeds germination (Figure 3a), suggesting that OsWD1 may be functional correlation with the seedling growth. Knockout of OsWD1 caused a shorter phenotype of transgenic rice seedlings (Figure 5a). On the contrary, overexpression of Os WD1 caused a taller phenotype that could be inhibited by external application of uniconazole to block GA biosynthesis (Figure 5a). Moreover, the expression of GA inducible genes, $O s E P 3 A$ and $\alpha$-amylase, were increased in OsWD1-Ox but decreased in OsWD1-Ri plants (Figure 6). These studies indicate that the role of OsWD1 is involved in GA biosynthesis and/or signaling pathways. There have been reported that GAs also play important roles in regulating seed development, and reproductive organ development, including both male and female flowers [38-40]. In agreement with the previous studies, we observed that the $O s W D 1$ overexpressing lines $(O x-1$ and $O x-2)$ produced large seeds, whereas the OsWD1 knockdown (Ri-1 and Ri-2) lines produced small seeds (Figure 5b), suggesting that OsWD1 might positively regulate grain size development in rice.

The OsWD1 shared a very high identity $(80 \%)$ on the amino acid sequence with a well known Arabidopsis TOPLESS (TPL) protein (Figure 2), we therefore suggest that OsWD1 might function similarly to TPL. Extensive studies have been demonstrated that TPL or TPR (TPLRELATED) play multiple roles for corepressors to modulate gene expression in diverse processes, such as auxin and jasmonic acid (JA) signaling, the flowering time, stress response, and gibberellic acid (GA) response [41-48]. With respect to GA, it is one of the most important regulators of many aspects of plant growth and development, and DELLAs are major plant growth repressors in repressing GA responses [49]. Studying the relationship between nonproteolytic regulation of DELLAs and GA signaling, Fukazaka et al. [47-48] found that under GA deficient condition, DELLA can interact with a transcription factor GAF1 (GAI-ASSOCIATED FACTOR 1), the resulting GAF1-DELLA complex acts as transcriptional activator to activate GA biosynthesis genes expression. In the presence of GA, dissociation of DELLA from GAF1 resulting in the binding of TPR to GAF1, the formation of GAF1-TPR complex plays a transcriptional repressor that feedback inhibition of specific target genes expression. These findings show that the TPR (TPL) seems to play a role as corepressor to inhibit biosynthesis of GA in response to GA signal. However, in contrast to these results, this study demonstrated that the TPL homolog, OsWD1, might act as a positive regulator for GA biosynthesis and/or response and enhanced seedling growth and seed size (Figures 5 and 6). The conflicting data on the regulation of GA biosynthesis in Arabidopsis TPR and rice OsWD1, may reflect homologous genes specific response as well as differences in plant species, which need to be addressed in the future studies.

\section{Conclusion}

The expression of $O s W D 1$ in germinating rice embryo is developmentally regulated. In gain- and loss-of-function studies, through ectopic gene-overexpressed or -silenced in transgenic rice plants, OsWD1 was shown to play a positive role in the seedling and seed development. Furthermore, the OsWD1 could enhance the expression of GA-inducible genes, $O s E P 3 A$ and $\alpha$-amylase, these results demonstrated that the $O s W D 1$ plays a role in increasing rice seedling growth might through a GA-dependent regulation pathway. These findings not only provide novel clues on the mechanisms of GA in seed development but also provide a strategy for improving rice grain size.

\section{Acknowledgements}

This work was supported by the grants from the Ministry of Science and Technology of the Republic of China (NSC92-2311-B-415-002 and MOST-104-2313-B-415-003-).

\section{References}

[1] E. J. Neer, C. J. Schmidt, R. Nambudripad, T. F. Smith "The ancient regulatory-protein family of WD-repeat proteins" Nature, vol. 371, pp. 297-300. 1994.

[2] S. van Nocker, P. Ludwig "The WD-repeat protein superfamily in Arabidopsis: conservation and divergence in structure and function" BMC Genomics, vol. 4, 50. 2003.

[3] J. Guo, J. G. Chen " $R A C K 1$ genes regulate plant development with unequal genetic redundancy in Arabidopsis" BMC Plant Biol, vol. 8, 108; doi:10.1186/1471-2229-8-108. 2008.

[4] E. A. Ananieva, G. E. Gillaspy, A. Ely, R. N. Burnette, F. L. Erickson "Interaction of the WD40 domain of a myoinositol polyphosphate 5-phosphatase with SnRK1 links inositol, sugar, and stress signaling" Plant Physiol, vol. 148, pp.18681882. 2008.

[5] T. F. Smith, C. Gaitatzes, K. Saxena, E. J. Neer "The WD repeat: a common architecture for diverse functions" Trends Biochem Sci, vol. 24, pp. 181-185. 1999.

[6] Y. Miyata, T. Shibata, M. Aoshima, T. Tsubata, E. Nishida "The molecular chaperone TRiC/CCT binds to the Trp-Asp 40 (WD40) repeat protein WDR68 and promotes its folding, protein kinase DYRK1A binding, and nuclear accumulation" $\mathrm{J}$ Biol Chem, vol. 289, pp. 33320-33332. 2014.

[7] J. C. Miller, W. R. Chezem, N. K. Clay "Ternary WD40 repeat-containing protein complexes: evolution, composition and roles in plant immunity" Front Plant Sci, vol. 6, 1108. 2016.

[8] X. Xu, I. Paik, L. Zhu, Q. Bu, X. Huang, X. W. Deng, E. Huq "PHYTOCHROME INTERACTING FACTOR1 enhances the E3 ligase activity of CONSTITUTIVE PHOTOMORPHOGENIC1 to synergistically repress photomorphogenesis in Arabidopsis" Plant Cell, vol. 26, pp. 1992-2006. 2014.

[9] K. A. Lease, J. Wen, J. Li, J. T. Doke, E. Liscum, J. C. Walker "A mutant Arabidopsis heterotrimeric G-protein $\beta$ subunit affects leaf, flower, and fruit development" Plant Cell, vol. 13, pp. 2631-2641. 2001. 
[10] S. Hoppins, L. Lackner, J. Nunnari "The machines that divide and fuse mitochondria" Annu Rev Biochem, vol. 76, pp. 751780. 2007.

[11] Z. Ben-Simhon, S. Judeinstein, T. Nadler-Hassar, T. Trainin, I. Bar-Ya'akov, H. Borochov-Neori, D. Holland "A pomegranate (Punica granatum L.) WD40-repeat gene is a functional homologue of Arabidopsis TTGland is involved in the regulation of anthocyanin biosynthesis during pomegranate fruit development" Planta, vol. 234, pp. 865-881. 2011.

[12] I. Mascheretti, R. Battaglia, D. Mainieri, A. Altana, M. Lauria, V. Rossi "The WD40-repeat proteins NFC101 and NFC102 regulate different aspects of maize development through chromatin modification" Plant Cell, vol. 25, pp. 404-420. 2013.

[13] X. W. Deng, M. Matsui, N. Wei, D. Wagner, A. M. Chu, K. A. Feldmann, P. H. Quail "COP1, an Arabidopsis regulatory gene, encodes a protein with both a zinc-binding motif and a $\mathrm{G}_{\beta}$ homologous domain" Cell, vol. 7, pp. 791-801. 1992.

[14] T. W. McNellis, K. U. Torii, X. W. Deng "Expression of an Nterminal fragment of COP1 confers a dominant-negative effect on light-regulated seedling development in Arabidopsis" Plant Cell, vol. 8, pp. 1491-1503. 1996.

[15] S. V. Nocker, P. Ludwig "The WD-repeat protein superfamily in Arabidopsis: conservation and divergence in structure and function” BMC Genomics, vol. 4, 50. 2003.

[16] R. A. Ach, P. Taranto, W. Gruissem "A conserved family of WD-40 proteins binds to the retinoblastoma protein in both plants and animals" Plant Cell, vol. 9, pp. 1595-1606. 1997.

[17] Y. W. Quian, Y. C. J. Wang, R. E. Hollingsworth, D. Jones, N. Ling, E. Y. H. P. Lee "A retinoblastoma-binding protein related to a negative regulator of Ras in yeast" Nature, vol. 364, pp. 648-652. 1993.

[18] Y. W. Quian, E. Y. H. P. Lee "Dual retinoblastoma-binding proteins with properties related to a negative regulator of Ras in yeast” J Biol Chem, vol. 270, pp. 25507-25513. 1995.

[19] R. Ruggieri, K. Tanaka, M. Nakafuku, Y. Kaziro, E. A. Toh, K. Matsumoto "MSI1, a negative regulator of the RAS-cAMP pathway in Saccharomyces cerevisiae" Proc Natl Acad Sci USA, vol. 86, pp. 8778-8782. 1989.

[20] M. Ohnacker, S. M. L. Barabino, P. J. Preker, W. Keller “The WD-repeat protein Pfs $2 p$ bridges two essential factors within the yeast pre-mRNA 3'-end-processing complex" EMBO J, vol. 19, pp. 37-47. 2000.

[21] R. P. Bhalerao, K. Salchert, L. Bakó, L. Ökrész, L. Szabados, T. Muranaka, Y. Machida, J. Schell, C. Koncz "Regulatory interaction of PRL1 WD protein with Arabidopsis SNF1-like protein kinases" Proc Natl Acad Sci USA, vol. 96, pp. 53225327. 1999.

[22] K. Sompornpailin, Y. Makita, M. Yamazaki, K. Saito “A WDrepeat-containing putative regulatory protein in anthocyanin biosynthesis in Perilla frutescens" Plant Mol Biol, vol. 50, pp. 485-495. 2002.

[23] Q. Tieu, V. Okreglak, K. Naylor, J. Nunnari “The WD repeat protein, Mdvlp, functions as a molecular adaptor by interacting with Dnm1p and Fis1p during mitochondrial fission” J Cell Biol, vol. 158, pp. 445-452. 2002.

[24] Y. Ouyang, X. Huang, Z. Lu, J. Yao, Ouyang et al. "Genomic survey, expression profile and co-expression network analysis of OsWD40 family in rice” BMC Genomics, vol. 13, 100. 2012.

[25] X. Gao, Z. Chen, J. Zhang, X. Li, G. Chen, X. Li, C. Wu "OsLIS-L1 encoding a lissencephaly type-1-like protein with WD40 repeats is required for plant height and male gametophyte formation in rice" Planta, vol. 235, pp. 713-727. 2012.

[26] Y. Kwon, S. I. Yu, J. H. Park, Y. Li, J. H. Han, H. Alavilli, J. I. Cho, T. H. Kim, J. S. Jeon, B. H. Lee "OsREL2, a rice TOPLESS homolog functions in axillary meristem development in rice inflorescence" Plant Biotechnol Rep, vol. 6, pp. 213-224. 2012.

[27] A. Yoshida, Y. Ohmori, H. Kitano, F. Taguchi-Shiobara, H. Y. Hirano "ABERRANT SPIKELET AND PANICLE1, encoding a TOPLESS-related transcriptional co-repressor, is involved in the regulation of meristem fate in rice" Plant $\mathrm{J}$, vol. 70, pp. 327-33. 2012.

[28] T. Murashige, F. Skoog "A revised medium for rapid growth and bioassays with tobacco tissue cultures" Physiol Plant, vol. 15, pp. 473-497. 1962.

[29] W. B. Bruce, A. H. Christensen, T. Klein, M. Fromm, P. H. Quail "Photoregulation of a phytochrome gene promoter from oat transferred into rice by particle bombardment" Proc Natl Acad Sci USA, vol. 86, pp. 9692-9696. 1989.

[30] S. L. Ho, W. F. Tong, S. M. Yu "Multiple mode regulation of a cysteine proteinase gene expression in rice" Plant Physiol, vol. 122, pp. 57-66. 2000.

[31] S. L. Ho, Y. H. Chao, W. F. Tong, S. M. Yu "Sugar differenti al and coordinately regulates gene expression via a complex signal transduction network and multiple control mechanisms" Plant Physiol, vol. 125, pp. 877-890. 2001.

[32] A. Marchler-Bauer, J. B. Anderson, C. DeWeese-Scott, N. D. Fedorova, L. Y. Geer, et al. "CDD: a curated Entrez database of conserved domain alignments" Nucleic Acids Res, vol. 31, pp. 383-387. 2003.

[33] I. Letunic, L. Goodstadt, N. J. Dickens, T. Doerks, J. Schultz, R. Mott, F. Ciccarelli, R. R. Copley, C. P. Ponting, P. Bork "Recent improvements to the SMART domain-based sequence annotation resource" Nucleic Acids Res, vol. 30, pp. 242-244. 2002.

[34] Z. N. Yang, X. R. Ye, J. Molina, M. L. Roose, T. E. Mirkov "Sequence analysis of a 282-kilobase region surrounding the citrus tristeza virus resistance gene (Ctv) locus in Poncirus trifoliate L. Raf' Plant Physiol, vol. 131, pp. 482-492. 2003.

[35] K. Gomi, M. Matsuoka "Gibberellin signalling pathway" Curr Opin Plant Biol, vol. 6, pp. 489-493. 2003.

[36] H. Itoh, M. Matsuoka, C. M. Steber "A role for the ubiquitin 26S-proteasome pathway in gibberellins signaling" Trends Plant Sci, vol. 8, pp. 492-497. 2003.

[37] S. Pattanaik, B. Patra, S. K. Singh, L. Yuan "An overview of the gene regulatory network controlling trichome development in the model plant, Arabidopsis" Front Plant Sci, vol. 5, 259. 2014.

[38] R. P. Pharis, R. W. King "Gibberellins and reproductive development in seed plants" Annu Rev Plant Physiol, vol. 36, pp. 517-568. 1985. 
[39] S. M. Swain, J. B. Reid, Y. Kamiya "Gibberellins are required for embryo and seed development in pea" Plant J, vol. 12, pp. 1329-1338. 1997.

[40] R. W. King, L. T. Evans "Gibberellins and flowering of grasses and cereals" Annu Rev Plant Biol, vol. 54, pp. 307328. 2003.

[41] J. A. Long, C. Ohno, Z. R. Smith, E. M. Meyerowitz "TOPLESS regulates apical embryonic fate in Arabidopsis" Science, vol. 312, pp. 1520-1523. 2006.

[42] Z. Liu, V. Karmarkar "Groucho/Tup1 family co-repressors in plant development" Trends Plant Sci, vol. 13, pp. 137-144. 2008.

[43] H. Szemenyei, M. Hannon, J. A. Long "TOPLESS mediates auxin-dependent transcriptional repression during Arabidopsis embryogenesis" Science, vol. 319, pp. 1384-1386. 2008.

[44] L. Pauwels, et al. "NINJA connects the co-repressor TOPLESS to jasmonate signaling" Nature, vol. 464, pp. 788791. 2010.

[45] B. Causier, M. Ashworth, W. Guo, B. Davies "The TOPLESS interactome: A framework for gene repression in Arabidopsis" Plant Physiol, vol. 158, pp. 423-438. 2012.

[46] Q. Tao, D. Guo, B. Wei, F. Zhang, C. Pang, H. Jiang, J. Zhang, T. Wei, H. Gu, L. J. Qu, G. Qina "The TIE1 transcriptional repressor links TCP transcription factors with TOPLESS/TOPLESS-RELATED corepressors and modulates leaf development in Arabidopsis" Plant Cell, vol. 25, pp. 421437. 2013.

[47] J. Fukazawa, H. Teramura, S. Murakoshi, K. Nasuno, N. Nishida, T. Ito, M. Yoshida, Y. Kamiya, S. Yamaguchi, Y. Takahashia "DELLAs function as coactivators of GAIASSOCIATED FACTOR1 in regulation of gibberellin homeostasis and signaling in Arabidopsis" Plant Cell, vol. 26, pp. 2920-2938. 2014.

[48] J. Fukazawa, T. Ito, Y. Kamiya, S. Yamaguchi, Y. Takahashi "Binding of GID1 to DELLAs promotes dissociation of GAF1 from DELLA in GA dependent manner" Plant Signal Behav, vol. 10, 10, e1052923. 2015.

[49] T. P. Sun, F. Gubler "Molecular mechanism of gibberellins signaling in plants" Annu Rev Plant Biol, vol. 55, pp. 197223. 2004. 